BULLETIN OF THE

AMERICAN MATHEMATICAL SOCIETY

Volume 81, Number 4, July 1975

\title{
FINITELY EMBEDDED MODULES OVER NOETHERIAN RINGS
}

BY S. M. GINN AND P. B. MOSS

Communicated by Barbara L. Osofsky, March 3, 1975

A right $R$-module is finitely embedded if it has finitely generated essential socle (see, e.g., Vamos [4]). In [1] Jategaonkar settled the Jacobson conjecture for left and right fully bounded noetherian rings by showing that every finitely generated finitely embedded module is artinian. It is an open question whether or not this property holds for an arbitrary left and right noetherian ring (though it is well known that right noetherian is not enough). We prove here that if $R$ is left and right noetherian and $M$ is a projective finitely generated finitely embedded module over $R$, then $M$ is artinian. This result can be extended to cover the case where $M$ is an arbitrary finitely embedded submodule of a finitely generated free $R$-module. The proof of this and related results will appear elsewhere. Even the case $M=R$ seems to be new and in this case we can obtain the more general

THEOREM. If $R$ is a left and right noetherian ring and if the right socle of $R$ is either left or right essential, then $R$ is artinian.

We note that there exist right noetherian rings with right essential right socle which are not right artinian (see, e.g., [2]).

All rings have identity and modules are unitary.

$l_{A}(X)$ and $r_{A}(X)$ denote, respectively, the left and right annihilators of $X$ in the ring $A$.

We require the following result of T. H. Lenagan [3] which is adapted to suit our purpose in the form of

LEMmA 1. Let $S, R$ be rings and let $R$ be right noetherian. Let $M$ be an (S-R)-bimodule such that ${ }_{S} M$ has finite length and $M_{R}$ is finitely generated. Then $R / r(M)$ is an artinian ring and $M_{R}$ has finite length.

We also need the following lemma whose proof is straightforward.

Lemma 2. Let $A$ be a subring of $B$ and suppose that $A$ is right

AMS (MOS) subject classifications (1970). Primary 16A46.

Copyright $\odot 1975$, American Mathematical Society 
noetherian and $B_{A}$ is finitely generated. Let $e$ be an idempotent in $B$ and $K_{A}$ an A-essential submodule of $(e B)_{A}$. Then $l_{e B e}(K)$ is nilpotent.

THEOREM 3 . Let $R$ be a left and right noetherian ring and let $T$ denote the full $(n \times n)$ matrix ring over $R$. If $e$ is an idempotent in $T$ and $(e T)_{R}$ is finitely embedded, then $(e T)_{R}$ is artinian.

Proof. Let $K$ denote the socle of $(e T)_{R}$. Now $T_{R}$ and $(e T)_{R}$ are noetherian and $e T$ is a left $e T e$ right $R$-bimodule. Since $T e T$ is left $T$-finitely generated it follows that $e T$ is left $e T e$-finitely generated.

If $I_{R}$ is a simple submodule of $(e T)_{R}$ and if $y \in T$, then either $e y I=0$ or $e y I$ is a simple $R$-submodule of $e T$. This shows that $e T e K$ is a subset of $K$ and so $K$ is an (eTe-R)-bisubmodule of $e T$. Since $K_{R}$ has finite length, the left-right reverse of Lemma 1 gives that the ring $e T e / l_{e T e}(K)$ is artinian. By Lemma $2 e T e$ is artinian. Now $e T$ has finite length as a left $e T e$-module, so by Lemma 1 again, $(e T)_{R}$ is artinian.

THEOREM 4. Let $R$ be a left and right noetherian ring and let $P$ be a finitely generated projective right $R$-module. If $P$ is finitely embedded then $P$ is artinian.

Proof. Suppose that $P$ has $n$ generators. The direct sum $P^{n}$ of $n$ copies of $P$ is $R$-isomorphic to an idempotently generated right ideal of the $(n \times n)$ matrix ring $T$ over $R$. Now $P^{n}$ is finitely embedded and therefore is artinian by Theorem 3. It follows that $P$ is artinian.

\section{REFERENCES}

1. A. V. Jategaonkar, Jacobson's conjecture and modules over fully bounded noetherian rings, J. Algebra 30 (1974), 103-121.

2. R. P. Kurshan, Rings whose cyclic modules have finitely generated socle, J. Algebra 15 (1970), 376-386. MR 41 \#5403.

3. T. H. Lenagan, Artinian ideals in noetherian rings (to appear).

4. P. Vamos, The dual of the notion of "finitely generated", J. London Math. Soc. 43 (1968), 643-646. MR 40 \#1425. ENGLAND

DEPARTMENT OF MATHEMATICS, BIRKBECK COLLEGE, LONDON W.C. 1, 